\title{
Post-concussion return to boxing protocol
}

\author{
N K Sethi, MD \\ Department of Neurology, New York Presbyterian Hospital, Weill Cornell Medical Center, New York, USA
}

Corresponding author: N K Sethi (sethinitinmd@hotmail.com)

Background: Concussion is a complex pathophysiological process affecting the brain, induced by traumatic biomechanical forces. Immediately following a concussion, an athlete is usually advised physical and cognitive rest until post-concussion symptoms abate. The athlete then enters a stepwise return-to-play protocol. Premature return to play risks a second concussion, second impact syndrome, exacerbation and persistence of post-concussive symptoms. Various sports governing organisations such as the National Football League have developed postconcussion return to play protocols.

Discussion: Professional boxing is a popular contact sport in which concussions are common. Professional boxing currently lacks a standardised post-concussion return to boxing protocol. Professional boxers are arbitrarily suspended for periods ranging from 30 to 90 days after suffering a technical knockout (TKO) due to multiple head and body shots or after a knockout (KO). For some boxers a neurology clearance is requested prior to their return to boxing.

Conclusion: Developing and implementing a postconcussion return to the boxing protocol will standardise a return to boxing decision-making process and help to protect a boxer's health. This paper proposes a postconcussion return to boxing protocol with the recommendation that the proposed protocol be debated vigorously by the scientific community and evidence-based guidelines be developed by the medical community in conjunction with the professional boxing governing bodies. Keywords: boxing, safety, concussion, return-to-play

S Afr J Sports Med 2016;28(2):61-62.DOI: 10.17159/2078-516X/2016/v28i2a464

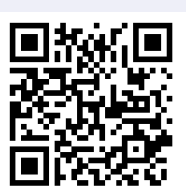

Concussion can be defined as a complex pathophysiological process affecting the brain, induced by traumatic biomechanical forces [1]. Concussion typically involves temporary impairment of neurological function with full recovery over a course of time. Currently there are no sensitive or specific biofluid (blood or cerebrospinal fluid) or imaging markers to identify and grade a concussion. In the absence of a valid and reliable biomarker of concussion, current emphasis is on early and accurate identification of the concussion, pulling the player out and advising cognitive and physical rest till symptoms abate. A systematic review of prognosis and return to play after sport concussion found no evidence on the effect of return-to-play guidelines on prognosis. The authors highlighted the need for well designed, confirmatory studies to better understand the consequences of sport concussion across different athletic populations and sports ${ }^{[2]}$.

\section{Defining a concussion in boxing}

Boxing is a unique contact sport in that every punch thrown at the head is thrown with the intention of causing a knockout (KO). No single definition of concussion is likely to be universally accepted in boxing and identifying mild grades of concussion (subconcussive blows) during the course of a boxing match is particularly challenging.

To help identify and manage a concussion in boxing the following recommendations are proposed based on evidence from other sports and clinical acumen:

1. A knock-down caused by punch to the head should raise concern for a concussion even if the boxer gets back to his feet before the count of 10 .

2. If a boxer is knocked down by a punch to the head and is unable to get up within the count of 10 (knockout), he should be automatically determined to have suffered a concussion and treated as such.

3. A boxer who suffers a technical knockout (TKO) on account of multiple head shots, should be assessed for concussion at the ringside and in the locker room.

4. A boxer who suffers multiple head shots during the course of the bout should be watched closely during the fight, assessed for concussion inbetween the rounds and after the fight is over.

5. A boxer who is determined to have suffered a concussion should not be allowed to box if he is symptomatic. Symptoms of concussion are varied and include a complaint of headache, nausea, double or blurred vision, disorientation and confusion, post-traumatic amnesia for events surrounding the fight, such as the name of the venue, name of the opponent, round in which $\mathrm{KO}$ or TKO occurred, impaired coordination, balance or gait ${ }^{[3]}$. Such a boxer should be assessed at the ringside or in the locker room for concussion with the aid of standardised tests such as Standardized Assessment of Concussion (SAC) test, Balance Error Scoring System (BESS) and the King-Devick test $[4,5,6]$. Such multimodal assessment for concussion improves diagnostic accuracy.

6. A boxer who is determined to have suffered a concussion should be closely watched in the locker room for any signs of neurological deterioration. The decision to transport the boxer to the nearest Level I trauma centre for evaluation (diagnostic imaging to rule out intracranial haemorrhage) should be made on a case- by-case basis.

7. Mandatory 90 day medical suspension should be issued at the venue for a KO caused by a head blow. This boxer then enters the post-concussion return to boxing protocol. For a technical knockout (TKO) caused by head and body blows, period of 
suspension ranges from 30 to 45 days. If the boxer, after evaluation by the ringside physician, is deemed to have suffered a concussion, the period of suspension may be longer on the discretion of the ringside physician. The boxer then enters the post-concussion return to boxing protocol.

8. A boxer who is determined to have suffered a concussion should be debarred from sparring or boxing until he is symptomatically back to baseline. He/she is instructed to see a physician (either a commission-appointed physician or an independent unaffiliated physician licensed in the state/country of residence of the boxer) within 24 hours of sustaining a concussion. The onus of enforcing this shall rest with the boxer, his/her corner and promoter.

The following post-concussion return to boxing protocol is proposed based on evidence from other sports and clinical acumen:

Once the boxer is symptom free for at least 24 hours and a release has been signed by the treating physician, he/she can begin a graded return to boxing as detailed below.

Day 1: Light aerobic activity (walking or stationary bike for 10 minutes, no resistance training)

Day 2: Sport-specific activity (jumping rope, shadow boxing)

Day 3: Non-contact training drills (Skill drills-pad work, speed bag, heavy bag)

Day 4: Full-contact practice (sparring with head gear)

Day 5: Return to boxing (return to competitive boxing occurs when the period of mandatory medical suspension has expired)

Each of the above steps should take 24 hours so that a boxer would take approximately one week to progress through the full post-concussion protocol once they are asymptomatic at rest. If any post- concussion symptoms occur while in the stepwise return to boxing programme, then the boxer should be instructed to return to the previous asymptomatic level and try to progress again after a further 24-hour period of rest has passed.

As good practice guidelines, the following are further suggested:

1. The referee and ringside physicians should be skilled in identifying concussions in the ring and in the management of a concussed boxer.

2. Establishing a database which tracks the number of concussions sustained by professional boxers. The goal of the database shall be to accurately record and report concussions sustained by an individual boxer, track the natural history of concussions and it is hoped this will be a valuable tool in the future for research on concussion risks, treatment and management. The goal and dream in the future is to have an online centralised concussion database for all professional boxers.
3. Every professional boxer should have a formal neuropsychological (neurocognitive) evaluation at the time of starting his professional career. This shall act as the baseline. The boxer should undergo a postinjury ImPACT test to document that neurocognitive function is back at baseline before clearance to return to competitive boxing (7).

4. Baseline neurocognitive evaluation should be repeated every two years. A decline in neurocognitive function should raise concern and be further assessed on a caseby-case basis.

5. Emphasis should be placed on educating boxers, trainers and coaches about concussions and the reported link between multiple concussions and chronic traumatic encephalopathy (CTE).

\section{Conclusion}

It is recommended that the above proposed protocol be debated vigorously by the scientific community and evidencebased guidelines be developed by the medical community in conjunction with professional boxing governing bodies. Boxing can be made safer and a standardised return to boxing protocol is very necessary.

\section{References}

1. Sharp DJ, Jenkins PO. Concussion is confusing us all. Pract Neurol 2015; 15(3):172-186. doi: 10.1136/practneurol-2015-001087

2. Cancelliere C, Hincapié CA, Keightley M, et al. Systematic review of prognosis and return to play after sport concussion: results of the International Collaboration on Mild Traumatic Brain Injury Prognosis. Arch Phys Med Rehabil 2014; 95(3 Suppl):S210-229. doi: 10.1016/j.apmr.2013.06.035

3. Lei-Rivera L, Sutera J, Galatioto JA, et al. Special tools for the assessment of balance and dizziness in individuals with mild traumatic brain injury. NeuroRehabilitation 2013; 32(3):463-472 doi: 10.3233/NRE-130869

4. Yengo-Kahn AM, Hale AT, Zalneraitis BH, et al. The Sport Concussion Assessment Tool: a systematic review. Neurosurg Focus 2016; 40(4):E6. doi: 10.3171/2016.1.FOCUS15611

5. Bell DR, Guskiewicz KM, Clark MA, et al. Systematic review of the balance error scoring system. Sports Health 2011; 3(3):287-295. doi: $10.1177 / 1941738111403122$

6. Galetta KM, Barrett J, Allen M, et al. The King-Devick test as a determinant of head trauma and concussion in boxers and MMA fighters. Neurology 2011; 76(17):1456-1462. doi 10.1016/j.jns.2011.07.039

7. Seifert T, Bernick C, Jordan B, et al. Determining brain fitness to fight: Has the time come? Phys Sportsmed 2015; 43(4):395-402 doi: 10.1080/00913847.2015.1081551 